\title{
Transient optical diffraction of GaN/aqueous interfaces: interfacial carrier mobility dependence on surface reactivity
}

\author{
Hoang Q. Doan, ${ }^{\dagger}$ Kevin L. Pollock, ${ }^{\dagger}$ and Tanja Cuk ${ }^{*,+, \dagger}$ \\ Department of Chemistry, University of California, Berkeley, Berkeley, CA, and Chemical \\ Sciences Division, Lawrence Berkeley National Laboratory, Berkeley, CA \\ E-mail: tanjacuk@berkeley.edu
}

\begin{abstract}
While charge transport and surface reactivity have thus far been treated as independent phenomena, the interfacial carrier mobility could be highly dependent on reaction intermediates that carry localized charge and can hop from site to site along the surface. Here, we demonstrate the use of surface sensitive transient optical grating spectroscopy to measure this lateral, interfacial carrier diffusivity at surfaces with different reactivity. We find that for $\mathrm{n}-\mathrm{GaN}$, for which substantial charge transfer occurs during equilibration with the water oxidation reaction, the interfacial hole diffusivity increases from air by a factor greater than two under $0.1 \mathrm{M} \mathrm{HBr}$ and $0.1 \mathrm{M} \mathrm{Na}_{2} \mathrm{SO}_{4}$ aqueous electrolytes.
\end{abstract}

KEYWORDS: diffusion coefficient, transient grating spectroscopy, surface states, charge transport

Heterogeneous catalytic activity at the solidelectrolyte interface involves the reaction itself and the transport of charge and reactant molecules to that interface. Charge transport

\footnotetext{
*To whom correspondence should be addressed

${ }^{\dagger}$ Department of Chemistry, University of California, Berkeley, Berkeley, CA

${ }^{\ddagger}$ Chemical Sciences Division, Lawrence Berkeley National Laboratory, Berkeley, CA
}

and surface reactivity are usually considered independent phenomena. ${ }^{1-3}$ Yet, charge transport within the solid, especially the lateral mobility along the interface, could be influenced significantly by mobile reaction intermediates that accommodate and transport charges along the surface. In a photo-electrochemical cell where the illuminated area is a small part of the total area of the catalytic surface, as is explored with nanoscale geometries, the lateral mobility of charge separated carriers could be especially important for promoting catalysis. ${ }^{4-7}$ This geometry is explicitly investigated in photoelectrochemical cells where a small area photovoltaic is connected to a much larger catalytic surface and leads to the highest solarto-hydrogen conversion efficiency to date $(18 \%$ by $^{8}$ and $25 \%$ by $^{9}$ ).

For the most part, the effects of an interface on charge carrier mobility have been investigated at the air interface, from which steady catalysis does not proceed. Carrier scattering from defect centers has been found to dominate, such that surface passivation, either through a capping layer or a chemical treatment, can directly increase the carrier mobility. ${ }^{10-12}$ More commonly, surface passivation leads to increased diffusion lengths in nanowires by limiting surface recombination rather than directly affecting carrier mobility. ${ }^{13-17}$

At the solid-electrolyte interface, from which catalysis can proceed, a different picture is ex- 
pected to guide interfacial carrier mobility, but has yet to be established. In cases where the surface mediates catalysis, which occurs readily at an aqueous electrolyte interface, reaction intermediates that carry localized charge, such as $\mathrm{Ti}-\mathrm{O}^{*}, \mathrm{Co}(\mathrm{IV})=\mathrm{O}$, and $\mathrm{Fe}(\mathrm{IV})=\mathrm{O}$, have been directly observed or calculated. ${ }^{18-21}$ These species can move from site to site, accompanied by proton coupled electron transfer (PCET). ${ }^{20}$ Their lateral mobility should be guided by the presence of neighboring sites, such as $\mathrm{M}-\mathrm{OH}$, to which they can easily transfer. In the semiconductor literature, these reaction intermediates and their neighboring sites are described as a narrow band of mid-gap surface states that mediate charge transfer and catalysis; ${ }^{22,23}$ carriers in this mid-gap band are expected to be mobile to the degree that there is a finite bandwidth. ${ }^{24}$

Here, we demonstrate the use of transient optical grating spectroscopy (TGS), where two interfering pump pulses create a diffraction grating from which a delayed probe diffracts, to measure the lateral diffusivity at semiconductor-electrolyte interfaces of different reactivity. While TGS has been applied to determine lateral diffusivities at the air interface of semiconductors such as Si, GaAs, and $\mathrm{GaN}^{25-29}$ and, additionally, at the $\mathrm{TiO}_{2}$ or GaAs/electrolyte interface ${ }^{30-34}$ to measure interfacial charge transfer, it has yet to extract diffusivities at the electrolyte interface.

Specifically, TGS is applied to investigate lateral hole diffusivity at the aqueous electrolyte interface of undoped GaN and n-GaN films. $\mathrm{GaN}$ is widely studied both experimentally and theoretically as a model for applications in catalysis because of its interesting surface chemistry. ${ }^{35-38}$ Nanowires and thin films of GaN have demonstrated catalytic activity for water oxidation in photo-catalysis. ${ }^{5,6,39}$ For GaN, during equilibration with an aqueous environment, interfacial mid-gap states that mediate catalysis are already generated at equilibrium, commonly referred to as Fermi level pinning. ${ }^{40,41}$ Therefore, reaction intermediates that carry localized charge occur even at open circuit, where the sample does not suffer from photo-corrosion and data taking is more facile. We find that the lateral, interfacial hole diffusivity increases by a

factor $>2$ from air at the n-GaN/aqueous electrolyte interface for both $\mathrm{pH}=1.4(0.1 \mathrm{M} \mathrm{HBr})$ and $\mathrm{pH}=7\left(0.1 \mathrm{M} \mathrm{Na}_{2} \mathrm{SO}_{4}\right)$. This increase in diffusivity is not sensitive to defect centers, since the surface recombination velocity, also obtained from the TGS kinetics, differs by almost an order of magnitude between the two electrolytes. In contrast to n-GaN, aqueous electrolytes do not change the interfacial hole diffusivity at the undoped GaN interface. For undoped GaN, the semiconductor Fermi level is close to the water oxidation potential and therefore, during equilibration, little charge transfer occurs and few reaction intermediates are created. Since the interfacial carrier mobility increases in the presence of reaction intermediates, the experiments for the first time reveal the positive correleation of interfacial charge transport with surface reactivity.
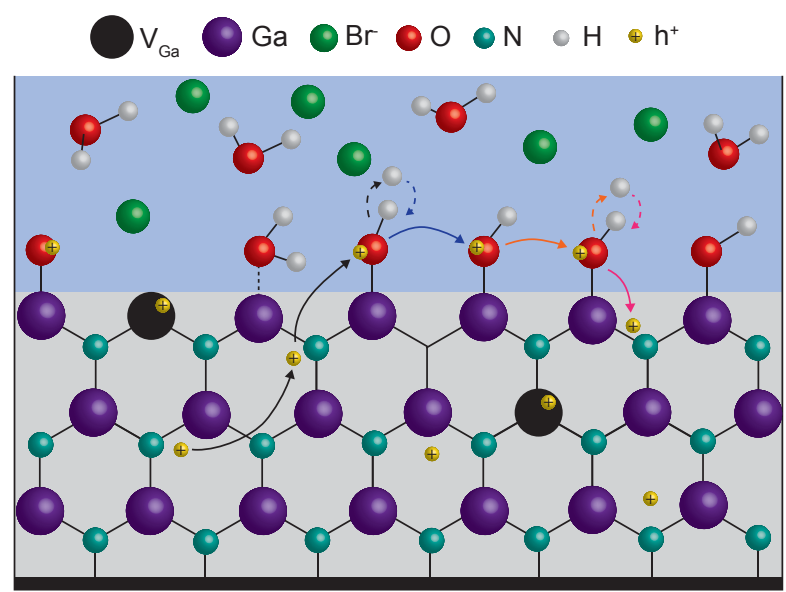

Figure 1: GaN(0001) surface with Ga sites terminated by $\mathrm{H}_{2} \mathrm{O}, \mathrm{OH}$, and $\mathrm{O}^{*}$ after equilibration with an aqueous $0.1 \mathrm{M} \mathrm{HBr}$ electrolyte. Holes from the nitrogen valence band hop on the surface (solid arrows), accompanied by proton release and capture (dotted arrows), before returning to the bulk.

The suggested origin for the increase in interfacial hole mobility is depicted schematically in Fig. 1. Charge transfer during equilibration at the $\mathrm{n}-\mathrm{GaN} /$ aqueous interface creates a surface which includes reaction intermediates that carry localized charge, such as $\mathrm{Ga}^{-} \mathrm{O}^{*}$, and a larger $\mathrm{Ga}-\mathrm{OH}$ population among $\mathrm{Ga}-\mathrm{H}_{2} \mathrm{O}$ adsorbates. These surface species comprise the 
interfacial mid-gap states specific to equilibra-

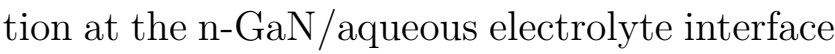
(as depicted in Fig. 2). Holes generated deep within the solid move along delocalized pathways within the nitrogen valence band, while the charge localized in reaction intermediates hops between neighboring (hydroxylated) sites, sometimes accompanied by PCET. Therefore, an additional current pathway for interfacial hole carriers is created by charge and proton hopping along the catalytic surface depicted, which should increase hole mobilites up to at least a diffusion length within the solid.

\section{Results and Discussion}

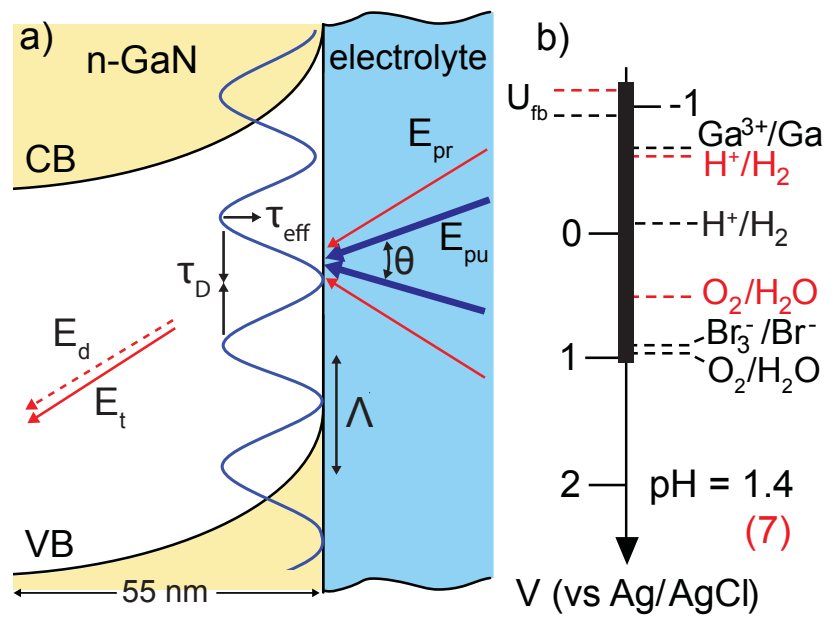

Figure 2: Transient grating spectroscopy setup. $\mathrm{E}_{p u}$ is the $266 \mathrm{~nm}$ pump beam. $\mathrm{E}_{p r}, \mathrm{E}_{t}$ and $\mathrm{E}_{d}$ are the incident, transmitted, and diffracted $800 \mathrm{~nm}$ probe beams, respectively. CB and VB are the conduction and valence band edge, respectively. $\theta$ and $\Lambda$ are the pump angle and grating spacing, respectively. b) Reduction potentials of $\mathrm{H}^{+} / \mathrm{H}_{2}, \mathrm{O}_{2} / \mathrm{H}_{2} \mathrm{O}, \mathrm{Ga}^{3+} / \mathrm{Ga}$ and $\mathrm{Br}_{3}^{-} / \mathrm{Br}^{-}$in solution at $\mathrm{pH}=1.4(7)$. The flatband potential $\left(\mathrm{U}_{f b}\right)$ at $\mathrm{pH}=1.4(7)$ is also indicated. The black block indicates the interfacial mid-gap band formed upon equilibration.

Shown in Fig. 2a is a representation of TGS that probes the diffusivity at the n-GaN/electrolyte interface. Two above bandgap (266 nm) interfering pump beams generate a diffraction grating of electron-hole carriers to within a penetration depth, $1 / \alpha=55 \mathrm{~nm}$, of the interface. The spatially modulated carrier grating with spacing $\Lambda$ depends on the pump angle, $\theta$, according to: $\Lambda=\frac{\lambda_{p u m p}}{2 \sin (\theta)}$. While the grating can decay via recombination and diffusion, the diffusive component decays with a time constant $\left(\tau_{D}\right)$ dependent on the grating spacing, $\Lambda$. The diffusivity is measured with the diffraction of a below bandgap (800 $\mathrm{nm}$ ) probe pulse in transmission mode using a phase-matched heterodynedetected boxcar geometry, as implemented previously. ${ }^{42-45}$ In both GaN and n-GaN, as will be discussed below, the lateral hole diffusivity in particular is extracted from the measured diffusivity.

The lateral hole diffusivity is measured at $\mathrm{pH}=1.4 \quad(0.1 \mathrm{M} \mathrm{HBr})$ and $\mathrm{pH}=7(0.1 \mathrm{M}$ $\mathrm{Na}_{2} \mathrm{SO}_{4}$ ) aqueous electrolyte interfaces in open circuit conditions. The appropriate Nernstian potentials are shown in Fig. 2b, along with the flatband potential $\left(\mathrm{U}_{f b}\right)$ for the $\mathrm{n}-\mathrm{GaN} /$ aqueous electrolyte interfaces measured by the MottSchottky capacitance (Supp. Fig. 1). Since $\mathrm{U}_{f b}$, the potential at which the Fermi level in the semiconductor is equal to the solution potential, is more negative than any of the Nernstian potentials, they will all proceed during equilibiration at the $\mathrm{n}-\mathrm{GaN}$ interface. The $\mathrm{Br}^{-} / \mathrm{Br}_{3}^{-}$reaction, occurring simultaneously with the $\mathrm{H}_{2} \mathrm{O} / \mathrm{O}_{2}$ reaction, suppresses the $\mathrm{Ga} / \mathrm{Ga}^{3+}$ photo-corrosion reaction, and is typically used as the electrolyte when demonstrating photo-catalytic water oxidation on GaN surfaces. ${ }^{6,46-48}$ SEM images, shown in Supp. Fig. 2, show minimal photo-corrosion at the GaN surface with the experimental pump fluence of $0.06 \mathrm{~mJ} \mathrm{~cm}^{-2}$. The $\mathrm{H}_{2} \mathrm{O} / \mathrm{O}_{2}$ and $\mathrm{Br}^{-} / \mathrm{Br}_{3}^{-}$reactions are expected to be mediated by interfacial mid-gap states, i.e. Fermilevel pinning, according to previous steady state and transient photo-electrochemical measurements ${ }^{40}$ and photo-luminescence in gas-phase environments. ${ }^{41}$

\section{Steady-State Photo-electrochemistry}

Two steady state, photo-electrochemical measurements establish the extent to which reactions at the n-GaN/aqueous interface are mediated through interfacial mid-gap states. First, 
the linear dependence of the photovoltage $\left(\mathrm{U}_{p h}\right)$ on the redox potential $\left(U_{\text {redox }}\right)$ of the solution, highlighted in Fig. 3a, exhibits a slope of 0.6. A slope of 1 implies no Fermi-level pinning. ${ }^{49} \mathrm{U}_{p h}$ is obtained from open circuit potential graphs (OCP) with and without excitation, as shown in Fig. 3b for the two electrolytes of interest (see Supp. Fig. 3 for the other two electrolytes), under the same pulsed laser conditions used for the diffusivity measurements.
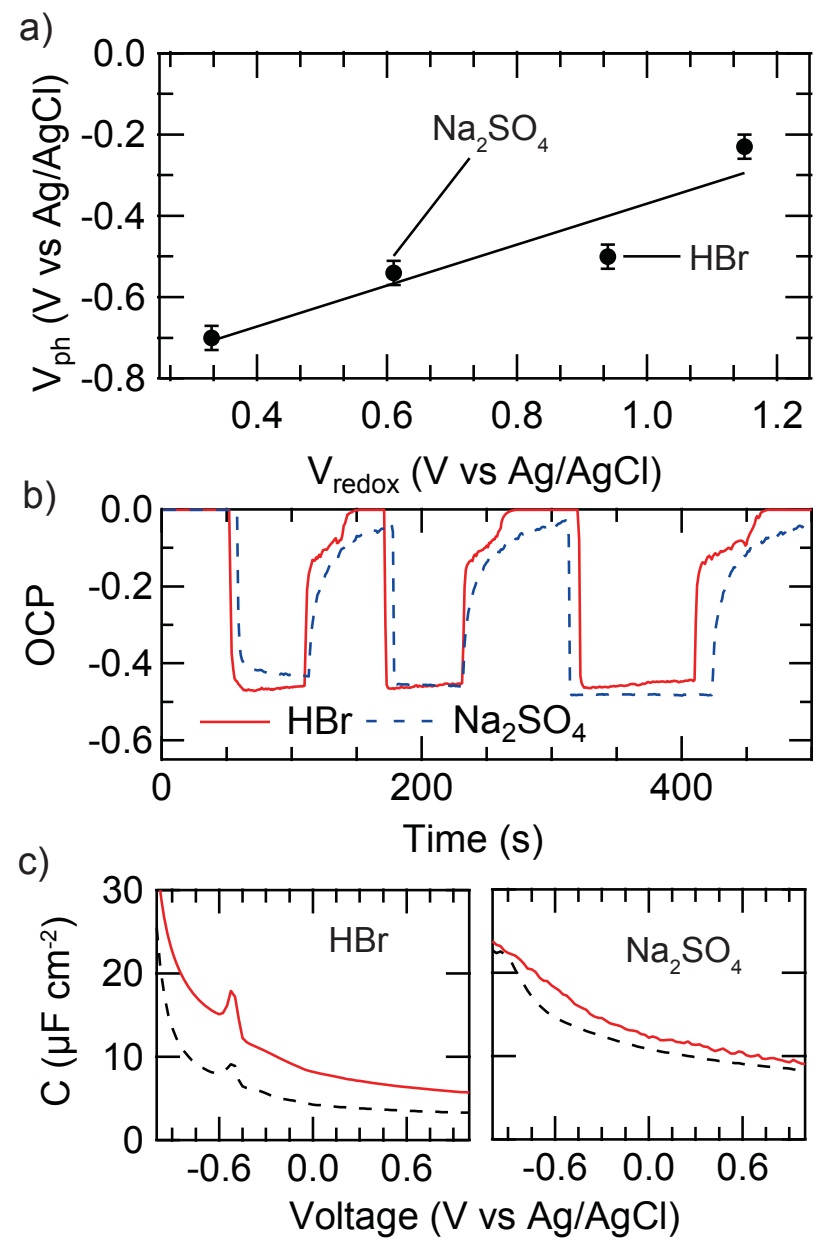

Figure 3: a) $\mathrm{V}_{p h}$, photovoltage, with respect to the Nernstian redox potential of various electrolytes. Solid line is linear fit with $d V_{p h} / d V_{\text {redox }} \sim 0.6 \mathrm{~b}$ ) Open circuit potential (OCP) over time for $0.1 \mathrm{M} \mathrm{HBr}$ (solid) and $\mathrm{Na}_{2} \mathrm{SO}_{4}$ (dotted) at light off and light on conditions. b) Capacitance with respect to applied potential in the dark (dotted) and illuminated (solid) for $0.1 \mathrm{M} \mathrm{HBr}$ and $\mathrm{Na}_{2} \mathrm{SO}_{4}$. Illuminated measurements were performed with 267 nm, $150 \mathrm{fs}, 1 \mathrm{kHz}$ pulsed light and only the illuminated area was exposed to the electrolyte.
Secondly, interfacial mid-gap states that pin the Fermi level are associated with an accumulated surface charge $\left(\mathrm{q}_{s s}\right)$. The surface charge develops as a result of charge transfer from surface species to the electrolyte, and along with the charge within the depletion layer of the semiconductor, $q_{s c}$, equilibrates the nGaN/electrolyte interface. It can be quantified using a combination of the steady state photovoltage measurements (Fig. 3b), Mott-Schottky capacitance measurements (Supp. Fig. 1), and a model of the n-type semiconductor/electrolyte interface that incorporates the several nanometer screening layer within the electrolyte, called the Helmholtz layer. While $\mathrm{q}_{s c}$ leads to a voltage drop across the depletion region of the semiconductor $\left(\mathrm{U}_{s c}\right)$, the voltage that drops across the Helmholtz layer $\left(\mathrm{U}_{H}\right)$ develops as a result of ions in solution screening both $\mathrm{q}_{s s}$ and $\mathrm{q}_{s c}$. The applied voltage required to reach $\mathrm{U}_{f b}$ is a sum of these two voltage drops. In a model used previously $^{23,50-53}$ and described in Supp. Fig. 4, $\mathrm{U}_{H}$ and $\mathrm{q}_{s s}$ are calculated using experimental inputs of $\mathrm{U}_{f b}$ and $\mathrm{U}_{s c}$, obtained from $V_{p h}$. With a Helmholtz layer assumed to be a parallel plate capacitor with $\mathrm{C}_{H} \sim 20 \mu \mathrm{F} \mathrm{cm}{ }^{-2}$, appropriate for aqueous solutions, ${ }^{54} \mathrm{U}_{H}$ and $\mathrm{q}_{s s}$ for the $\mathrm{n}$ GaN/aqueous electrolyte interfaces are 70-80\% of $\mathrm{U}_{f b}$ and $\sim 10^{14}$ charges $\mathrm{cm}^{-2}$ (Supp. Fig. 4). In this model and at an interface where $\mathrm{U}_{f b}$ is negative of all the Nernstian potentials, the surface charge is thought of as positive.

While the actual surface charge with distance from the electrode requires a more detailed description, ${ }^{55}$ it can still be used as a metric to differentiate surfaces during equilibration. ${ }^{22}$ A $\mathrm{q}_{s s}$ of $10^{14} \mathrm{~cm}^{-2}$ is a substantial surface charge that differentiates the $\mathrm{n}-\mathrm{GaN} /$ aqueous interface from the undoped $\mathrm{GaN} /$ aqueous, undoped GaN/air, and n-GaN/air interfaces at open circuit. The surface charge far exceeds the integrated charge within the depletion layer $\left(\mathrm{q}_{s c}\right.$ of $\left.10^{12} \mathrm{~cm}^{-2}\right)$ that would occur similarly at the n-GaN/air and n-GaN/electrolyte interfaces, since surface adsorbed water species are common to both; ambient pressure photoemission of the lightly doped n-GaN/water adsorbed surface finds that the surface band bending is fairly insensitive to the water coverage. ${ }^{56}$ 
The n-GaN/air interface, due to the lack of a Helmholtz screening layer, can only support the charge transferred by the depletion layer, or $\sim$ $10^{12} \mathrm{~cm}^{-2} .{ }^{22}$ For the undoped GaN/air and undoped GaN/aqueous interfaces, the Fermi level of the bulk semiconductor is close to the water oxidation potential, according to GaN photoluminescence studies that place the surface states close to the middle of the band gap. ${ }^{41}$ Therefore, much less charge is transfered during equilibration than for n-GaN, such that neither a substantial depletion layer or surface charge (for the aqueous interface) is generated.

Surface charge at the electrolyte interface can accumulate as a result of equilibration with the solution, as described above, or due to excess photo-holes induced by the laser pulse. However, the capacitance measurements, $\mathrm{C}(\mathrm{V})$, shown in Fig. 3c, reveal no significant differences in the voltage dependence between dark and laser illuminated $\left(0.06 \mathrm{~mJ} / \mathrm{cm}^{2}\right)$ conditions. This is unlike at aqueous electrolyte interfaces with highly doped $\mathrm{SrTiO}_{3}, \mathrm{Si}$, and $\mathrm{InP}$ where large light induced changes occur in $\mathrm{C}(\mathrm{V})$ at similar fluences. ${ }^{50,57,58}$ Therefore, the TGS technique employed acts as a weak probe for the interfacial carrier diffusivity, without substantially altering the surface species during the measurement.

\section{Diffraction Efficiency}

A simplified model of the diffraction efficiency decay is $\eta(t) \propto \exp \left(-2 \mathrm{t} \tau^{-1}\right)$. The diffusivity, $D$, from TGS kinetics can be determined using a fit to a monoexponential decay along with eq. 1.

$$
\tau^{-1}=\frac{1}{\tau_{D}}+\frac{1}{\tau_{e f f}}=D \frac{4 \pi^{2}}{\Lambda^{2}}+\frac{1}{\tau_{e f f}}
$$

The grating decay rate $\left(\tau^{-1}\right)$ has a lateral, diffusive decay component $\left(\tau_{D}\right)$ controlled by the grating spacing, $\Lambda$, and a component given by the spatially uniform excitation at large $\Lambda$ $\left(\tau_{\text {eff }}\right) . \quad \tau_{\text {eff }}$ is the excited state lifetime of bulk and surface recombination/trapping processes that decrease the diffusive carrier population. Given that a narrower carrier concentration gradient develops with smaller $\Lambda, \tau_{D}^{-1}$ is expected to increase as $\Lambda$ decreases or $\mathrm{q}^{2}=$
$4 \pi^{2} / \Lambda^{2}$ increases. By plotting $\tau^{-1}$ as a function of $\mathrm{q}^{2}, D$ and $\tau_{\text {eff }}$ are obtained from the slope and intercept, respectively, of a best-fit line. Additionally, the TGS kinetics can be modeled using the full second-order differential equation for diffraction efficiency as derived in Supp. Fig. 5 which, using the D obtained from the linear fit, also defines the surface recombination velocity, $S$ and bulk carrier recombination, $\tau_{R} \cdot{ }^{29}$

The extent to which the TGS provides the ambipolar diffusion coefficient, $\mathrm{D}_{a}$ or is a direct measurement of the hole diffusion coefficient, $\mathrm{D}_{h}$ depends on the injected carrier concentrations $\left(\Delta N_{e}, \Delta N_{h}\right)$, the electron and hole diffusion coefficient $\left(\mathrm{D}_{e}, \mathrm{D}_{h}\right.$, respectively) and the doping of the sample $\left(n_{0}\right)$, is given by ${ }^{59}$

$$
D=\frac{\left(n_{0}+\Delta N_{e}+\Delta N_{h}\right) D_{e} D_{h}}{\left[\left(n_{0}+\Delta N_{e}\right) D_{e}+\left(\Delta N_{h}\right) D_{h}\right]}
$$

According to Scajev et. al., two regimes exist for eq. 2 as noted by a steep sigmoidal relationship between $\mathrm{D}$ and injected carrier density for undoped GaN (Fig. 3a of Scajev et. al.). ${ }^{25}$ The steep sigmoidal relationship implies that within an order of magnitude of tuning $\Delta N$ (or $\mathrm{n}_{0}$ ), one of two regime is accessed. For a moderate/high injection regime where $\Delta N_{e}=\Delta N_{h} \gg \mathrm{n}_{0}$, the carrier diffusivity behaves as ambipolar $D_{a} \approx \frac{2 D_{h} D_{e}}{D_{h}+D_{e}}$. On the other hand when $\Delta N_{e}=\Delta N_{h} \ll \mathrm{n}_{0}$, the low injection regime is reached and the carrier diffusivity $D$ behaves as $D_{h}$.

The pump fluence used $\left(\sim 0.06 \mathrm{~mJ} \mathrm{~cm} \mathrm{~cm}^{-2}\right.$ or $10^{14}$ carriers $\mathrm{cm}^{-2}$ ) is below that at which bimolecular (radiative) and auger recombination become important in GaN, such that the above analysis can be employed and a monoexponential fit can be used to extract $D .{ }^{25}$ This pump fluence initially leads to a carrier density of $10^{19}$ carriers $\mathrm{cm}^{-3}$ within the $55 \mathrm{~nm}$ penetration depth. However, the carrier density is significantly diluted by diffusion into the bulk during the measurement, as will be discussed below. While diffusion into the bulk occurs, the diffusive component extracted from the TGS kinetics, $\tau_{D}^{-1}$, measures the lateral diffusion since the transimitted probe integrates the excited carrier density along the sample depth. ${ }^{26,60}$ 
In the following experiments, $\Lambda$ is varied from 12 to $2.3 \mu \mathrm{m}$ in air and, due to the change in refractive index in water, 16 to $3.1 \mu \mathrm{m}$ in aqueous electrolyte solution (Supp. Fig. 6). The monoexponential fits of the data to extract $\tau^{-1}$ for each grating spacing are shown in Supp. Fig. 7. Monoexponential fits were started at $3 \mathrm{ps}$, after both thermalization and charge separation (for n-doped samples) are expected to occur. Error bars are obtained using the standard error of three trials to get 4\% (Supp. Fig. 8). The fits of the TGS kinetics shown in Fig 4 are that of the full TGS differential equation, with the input of $\mathrm{D}$ from the monoexponential fits.

\section{Diffusivity at GaN/electrolyte in- terfaces}

Figure 4 demonstrates the variation in the interfacial diffusivity at undoped GaN and n-GaN interfaces with air and electrolyte. Based on the linear fit of $\tau^{-1}$ vs. $q^{2}$, undoped GaN has a $\mathrm{D}$ of $1.4 \pm 0.1 \mathrm{~cm}^{2} \mathrm{~s}^{-1}$ in air, as shown in Fig. 4a. For undoped $\mathrm{GaN}, \mathrm{n}_{0} \sim 10^{15} \mathrm{~cm}^{-3}$ and $\Delta N \sim 10^{19} \mathrm{~cm}^{-3}$. Therefore, the moderate/high excitation regime of eq. 2 is accessed. Since $D_{h} \ll D_{e}$ for GaN, TGS measures $D_{a} \approx 2 D_{h} .{ }^{61} D_{a}$ is comparable to previous values obtained by TGS experiments on GaN. ${ }^{25,26,60}$ From $\mathrm{D}_{h}$, the hole mobility determined by Einstein's relation, $\mu_{h}=D_{h}\left(\frac{k_{B} T}{e}\right)^{-1}$, is $27 \mathrm{~cm}^{2} \mathrm{~V}^{-1} \mathrm{~s}^{-1}$. For comparison, the reported bulk value for undoped and n-GaN ranges from $300-5 \mathrm{~cm}^{2} V^{-1} \mathrm{~s}^{-1,62-65}$ a bulk $\mu_{h}$ of $\sim$ $10 \mathrm{~cm}^{2} V^{-1} \mathrm{~s}^{-1}$ has been found for majority holes in p-doped GaN. ${ }^{66,67}$

When undoped GaN is introduced to an electrolyte environment, i.e. $0.1 \mathrm{M} \mathrm{HBr}$, no significant change in diffusivity was observed. As shown in Fig $4 \mathrm{a}, D_{a}$ remains $1.4 \pm 0.6 \mathrm{~cm}^{2} \mathrm{~s}^{-1}$. The inset shows the kinetics do not change between air and $0.1 \mathrm{M} \mathrm{HBr}$ for both the largest and smallest $\Lambda$. Importantly, the data exhibits no substantial difference in kinetics for the range of grating spacings measured, as can be seen by comparing Fig. 4b to Fig. 4c.

For n-GaN, the diffusivity is substantially different from undoped $\mathrm{GaN}$ at the air interface. Here, D is $0.8 \pm 0.1 \mathrm{~cm}^{2} \mathrm{~s}^{-1}$ in air (Fig. $4 \mathrm{~d}$ ) from the linear fit. Unlike in undoped GaN, the TGS of $\mathrm{n}-\mathrm{GaN}$ approaches the low injection limit due to the increased $n_{0} .{ }^{25}$ Although the calculated injection density at the earliest time scales, $\Delta N\left(10^{19} \mathrm{~cm}^{-3}\right)$ is similar to $\mathrm{n}_{0}\left(5 \times 10^{18} \mathrm{~cm}^{-3}\right)$, two experimental considerations suggest a low injection limit. First, the effective injected carrier density at times where the diffusive decay manifests, namely around 200-400 ps, can be 5-10 times lower than the initial $\Delta N$ reported for GaN because of changes to the carrier density profile by diffusion and recombination into the bulk. ${ }^{29,68,69}$ Second, the TGS kinetics are independent of pump fluences which should change the carrier injection by an order of magnitude (Supp. Fig. 9). Additionally, the diffusivity obtained experimentally for $\mathrm{n}$-GaN is nearly equivalent to the $\mathrm{D}_{h}$ obtained for undoped GaN, which is the expected value of the low injection limit (i.e. half the ambipolar diffusivity). Taken together, the experimental considerations and the measured values of the carrier diffusivity demonstrate the TGS of n-GaN directly probes the hole carrier diffusivity $\left(\mathrm{D} \sim D_{h}\right)$.

A significant increase in $\mathrm{D}_{h}$ to $2.1 \pm 0.3 \mathrm{~cm}^{2}$ $\mathrm{s}^{-1}$ is observed at the $\mathrm{n}-\mathrm{GaN} / 0.1 \mathrm{M} \mathrm{HBr}$ electrolyte interface, based on the linear fit of $\tau^{-1}$ vs $q^{2}$ (Fig. 4d). The change in kinetics between air and 0.1 M HBr environments is highlighted at the smallest and largest grating spacing in the inset of the Fig. 4d. The data over a range of grating spacings shows significantly faster kinetics in electrolyte (Fig. 4f) compared to air (Fig. 4e). Taken together, the data and analysis show that $\mathrm{D}_{h}$ increases at the $0.1 \mathrm{M} \mathrm{HBr}$ interface by a factor of 2.6 from the air interface. 
Table 1: Variables obtained from fits using diffraction efficiency model

\begin{tabular}{|l|l|c|r|r|}
\hline Sample & $\mathrm{S}\left(\frac{\mathrm{cm}}{\mathrm{s}}\right)$ & $\mathrm{D}_{h}\left(\frac{\mathrm{cm}}{\mathrm{s}}\right)$ & $\tau_{R}(\mathrm{ps})$ & $\mathrm{L}_{p}(\mathrm{~nm})$ \\
\hline $\mathrm{GaN}$ & $4.0 \times 10^{3}$ & 0.7 & 407 & 169 \\
$\mathrm{GaN}(\mathrm{HBr})$ & $7.0 \times 10^{3}$ & 0.7 & 389 & 165 \\
n-GaN & $1.6 \times 10^{4}$ & 0.8 & 1000 & 283 \\
n-GaN $(\mathrm{HBr})$ & $5.0 \times 10^{3}$ & 2.1 & 505 & 326 \\
n-GaN $\left(\mathrm{Na}_{2} \mathrm{SO}_{4}\right)$ & $2.5 \times 10^{4}$ & 1.8 & 703 & 356 \\
\hline
\end{tabular}

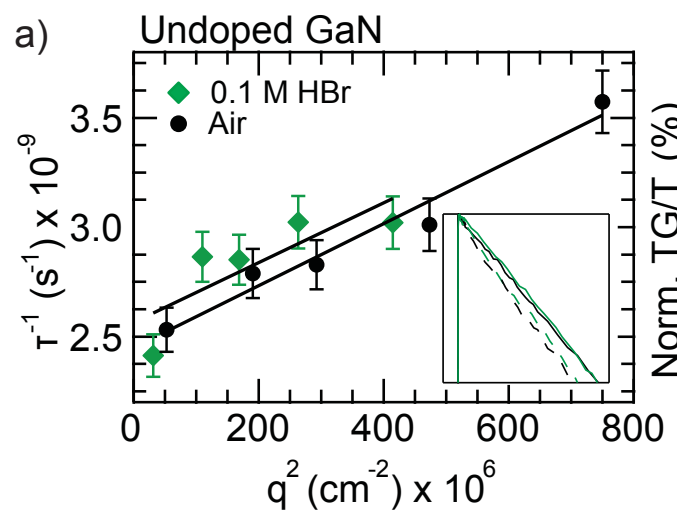

b) Air

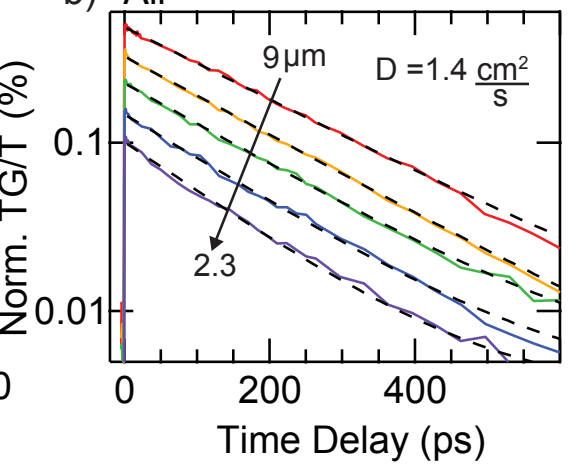

d)

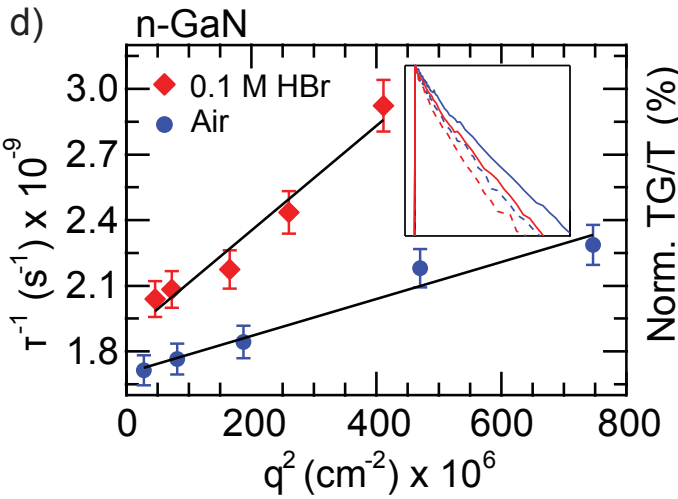

e) Air

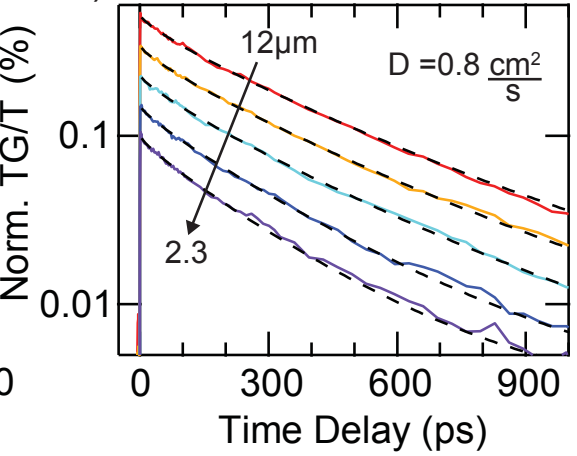

c) $0.1 \mathrm{M} \mathrm{HBr}$

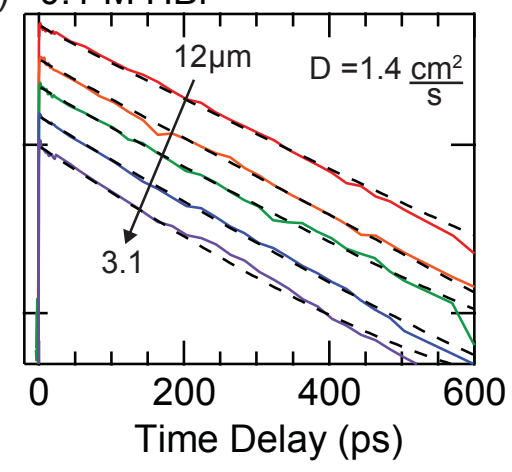

f) $0.1 \mathrm{M} \mathrm{HBr}$

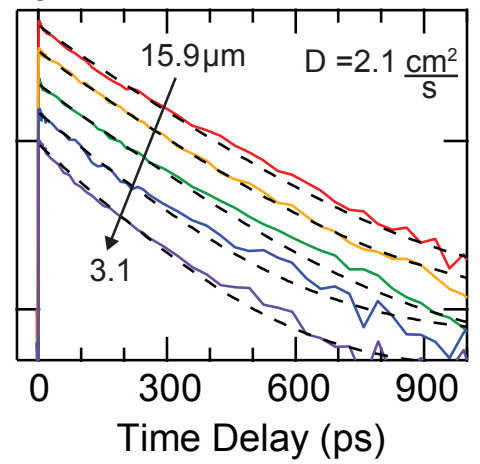

Figure 4: Decay rates $\left(\tau^{-1}\right)$ as a function of $\mathrm{q}^{2}$ for a) undoped GaN and d) n-GaN in air (circles) and in $0.1 \mathrm{M} \mathrm{HBr}$ (diamonds). Slope and intercept obtained from linear fit gives $\mathrm{D}=1.4 \pm 0.1 \mathrm{~cm}^{2}$ $\mathrm{s}^{-1}$ and $407 \pm 10 \mathrm{ps}$ in air and $\mathrm{D}=1.4 \pm 0.6 \mathrm{~cm}^{2} \mathrm{~s}^{-1}$ and $389 \pm 19 \mathrm{ps}$ in $0.1 \mathrm{M} \mathrm{HBr}$, respectively, for undoped GaN. D = $0.8 \pm 0.1 \mathrm{~cm}^{2} \mathrm{~s}^{-1}$ and $585 \pm 12 \mathrm{ps}$ in air and D $=2.1 \pm 0.3 \mathrm{~cm}^{2} \mathrm{~s}^{-1}$ and $505 \pm 16 \mathrm{ps}$ in $0.1 \mathrm{M} \mathrm{HBr}$, respectively, for n-GaN. Error bars represent standard error of fit. Insets: Comparison of normalized TG/T (\%) kinetics for largest (solid) and smallest (dashed) grating spacing in air and $0.1 \mathrm{M} \mathrm{HBr}$. Normalized TG/T (\%) kinetics (solid) for each grating spacings with TGS differential equation model fits (dashed) for b) undoped GaN in air, c) undoped GaN in 0.1 M HBr, e) n-GaN in air and f) n-GaN in 0.1 M HBr. Offsets are added for clarity. 


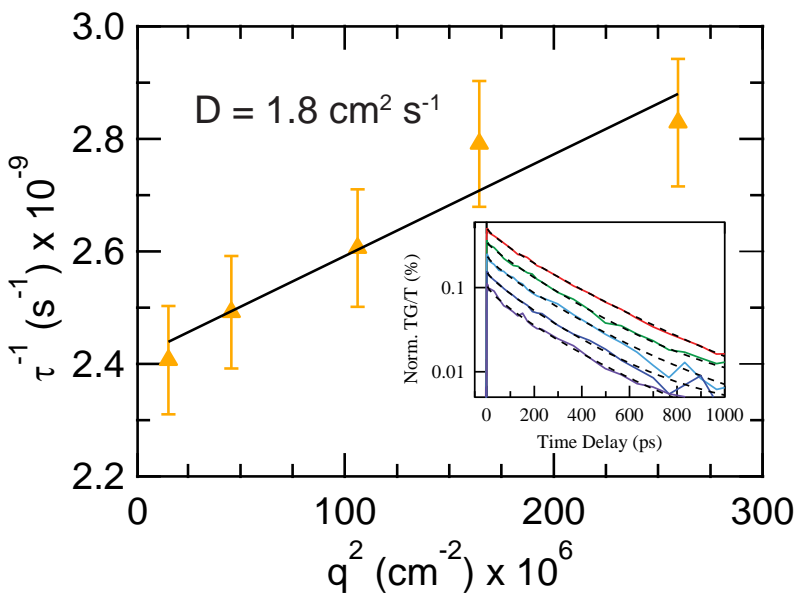

Figure 5: Decay rates $\left(\tau^{-1}\right)$ as a function of $\mathrm{q}^{2}$ for $n-G a N$ in $0.1 \mathrm{M} \mathrm{Na}_{2} \mathrm{SO}_{4}$. Slope and intercept obtained from linear fit gives $\mathrm{D}=1.8 \pm 0.3$ $\mathrm{cm}^{2} \mathrm{~s}^{-1}$ and $415 \pm 8 \mathrm{ps}$ in $0.1 \mathrm{M} \mathrm{Na}_{2} \mathrm{SO}_{4}$, respectively. Inset: Normalized TG/T (\%) kinetics (solid) for each grating spacings with TGS differential equation model fits (dashed) for nGaN in $0.1 \mathrm{M} \mathrm{Na} \mathrm{NO}_{4}$. Offsets are added for clarity.

The increased hole diffusivity at the nGaN/aqueous interface is not unique to an $\mathrm{HBr}$ electrolyte at $\mathrm{pH}=1.4$, but also occurs with an $\mathrm{Na}_{2} \mathrm{SO}_{4}$ electrolyte at $\mathrm{pH}=7$, as shown in Fig. 5. For $0.1 \mathrm{M} \mathrm{Na}_{2} \mathrm{SO}_{4}, \mathrm{D}_{h}$ is $1.8 \pm$ $0.3 \mathrm{~cm}^{2} \mathrm{~s}^{-1}$, a factor of 2.25 increase from air. While the addition of the $\mathrm{Br}^{-} / \mathrm{Br}_{3}^{-}$redox potential and a change in $\mathrm{pH}$ does affect the interfacial hole diffusivity, it is not substantially altered. That is, in both cases, equilibration to the $\mathrm{H}_{2} \mathrm{O} / \mathrm{O}_{2}$ redox potential is what creates the unique $\mathrm{n}-\mathrm{GaN} /$ aqueous electrolyte interface that changes the lateral diffusivity. The lack of a pronounced difference between the two electrolytes in terms of the overall surface composition is reflected in the similar behavior of the photovoltage (Fig. 3) and that both interfaces lead to $\mathrm{q}_{s s} \sim 10^{14} \mathrm{~cm}^{-2}$.

While it is expected that the dominant composition of the surface in the presence of aqueous electrolytes will be a mixture of surface water species, the two electrolyte interfaces could have substantially different defect densities. Using the full TGS differential equation model mentioned above, shown as fits on the TGS kinetics of Fig 4, and detailed in the sup- plementary, this defect density can be quantified by the surface recombination velocity, S, reported in Table 1. A surface that is well passivated, i.e. low defect density, has a low $\mathrm{S}$ while a surface with many recombination centers has a high S. For undoped GaN, S $=4 \times 10^{3} \mathrm{~cm} \mathrm{~s}^{-1}$ which agrees with previous experiments. ${ }^{70} \mathrm{No}$ change is observed when an aqueous electrolyte environment is introduced. For $n-\mathrm{GaN} / \mathrm{HBr}$, the aqueous electrolyte environment does significantly effect the surface passivation, leading to almost an order of magnitude decrease in $\mathrm{S}$ from $\sim 10^{4} \mathrm{~cm} \mathrm{~s}^{-1}$ in air to $\sim 10^{3} \mathrm{~cm} \mathrm{~s}^{-1}$. On the other hand, for the $n-\mathrm{GaN} / \mathrm{Na}_{2} \mathrm{SO}_{4}$ aqueous interface, the $\mathrm{S}$ remains similarly high as at the air interface. This suggests that, while the defect density and surface recombination velocity are sensitive to the specific counter ions and $\mathrm{pH}$ of the aqueous electrolyte, the interfacial hole diffusivity is not. The substantially lower $\mathrm{S}$ determined for $\mathrm{HBr}$ is likely related to the lower photo-corrosion observed during catalysis, as compared to $\mathrm{Na}_{2} \mathrm{SO}_{4}$. The lower S suggests $\mathrm{HBr}$ mediates reactions that avoid trapping holes in defect sites of GaN, thought to create $\mathrm{Ga}^{+3} \cdot{ }^{47,71,72}$

The increase in the lateral hole diffusivity occurs uniquely at the n-GaN/aqueous electrolyte interface, which leads to insights into its origin. When the $\mathrm{n}-\mathrm{GaN} /$ aqueous electrolyte interface equilibrates with the water oxidation reaction, a surface charge develops simultaneously with a depletion layer in the semiconductor and a Helmholtz screening layer at the interface. On the other hand, at the n-GaN/air interface, a depletion layer and at the undoped GaN/electrolyte interface, a Helmholtz screening layer, will develop separately and without a substantial surface charge. However, at both interfaces, the hole diffusivity remains $\sim 0$.7$0.8 \mathrm{~cm}^{2} \mathrm{~s}^{-1}$ (Table I). Therefore, the change in the hole diffusivity that occurs at the nGaN/aqueous interface is attributed to a surface charge that modifies the composition of surface water species. This finding is further substantiated by the fact that the interfacial hole diffusivity is sensitive to the overall composition of surface water species, and not specific defect centers, since $\mathrm{S}$ changes largely between 
the two electrolytes and D does not.

The surface charge generated at the nGaN/aqueous interface should modify surface water species by creating reaction intermediates and changing the distribution of hydroxylated and water adsorbed sites, as depicted in Fig. 1. An GaN (0001) surface with a monolayer of adsorbed water, calculated by DFT, leads to a distribution of $\mathrm{Ga}-\mathrm{OH}$ and $\mathrm{Ga}-\mathrm{H}_{2} \mathrm{O}$ sites. ${ }^{35,73}$ During equilibration at the $\mathrm{n}-\mathrm{GaN} /$ aqueous interface, the distribution of these surface water species will alter, and could contain a significant population of $\mathrm{Ga}-\mathrm{OH}$ and $\mathrm{Ga}-\mathrm{O}^{*}$, as suggested by ambient pressure photoemission of the nGaN (0001) surface exposed to water ${ }^{56}$ and by calculation of time scale evolutions of excited holes at different GaN surfaces. ${ }^{74}$ At $300 \mathrm{~K}$, phonon-assisted charge carrier hopping accompanied by PCET is expected between sites. ${ }^{20}$ Therefore, the equilibrated surface, that includes both localized charge in the form of reaction intermediates and neighboring sites to which the charge can easily hop (e.g. Ga-OH) should increase surface hopping mobility. In the energy band description of this interface, equilibration increases the density of surface states and broadens the mid-gap band ${ }^{75,76}$ of the $\mathrm{n}-\mathrm{GaN} /$ aqueous interface with respect to the undoped GaN/air, undoped GaN/aqueous, and n-GaN/air interfaces. Surface hopping could affect carrier mobility for many lattice lengths into the bulk for two reasons: (1) The surface states form a narrow, mid-gap band such that energy shifts in the photoluminescence reflect their filling with $\mathrm{pH}$, as observed on $\mathrm{GaN}^{41}$ and (2) Diffusion lengths are $\sim 200$ $\mathrm{nm}$ in GaN (Table I).

The increased interfacial carrier mobility can be considered one step in the catalytic process that creates a current pathway at the solidliquid interface. One way to quantify the opening of this current pathway is through the mean square displacement of the charge carrier profile from the average position of the initial profile, $<x^{2}>(\mathrm{t})$, along the air vs. electrolyte interface. The mean square displacement, according to $\left\langle x^{2}\right\rangle \sim D t$, increases by the same factor as the diffusivity, or by 2.6 at the $n-G a N / H B r$ interface. The standard deviation $\left(\sqrt{\left.<x^{2}\right\rangle}\right)$ of the carrier profile is $60 \%$ higher and hence, a $60 \%$ larger interfacial distance is traversed at the electrolyte interface. Another way to quantify the opening of the current pathway, especially important for photo-catalysis, is the hole diffusion length, $L_{p}=\sqrt{D_{h} \tau_{R}}$. The hole diffusion lengths $\left(L_{p}\right)$ for the different interfaces are shown in Table 1. For undoped GaN in air and in aqueous electrolyte, $L_{p}$ is $\sim 200 \mathrm{~nm}$ in agreement with previous experiments. ${ }^{65} L_{p}$ increases by $\sim 100 \mathrm{~nm}$ for $\mathrm{n}-\mathrm{GaN}$, but mostly due to the increased recombination time $\tau_{R}$. On the other hand, an additional $\sim 50 \mathrm{~nm}$ is observed when an aqueous electrolyte is introduced to the nGaN surface and is due to the increased diffusivity, since $\tau_{R}$ lowers. This demonstrates that at the n-GaN/electrolyte interface, photo-holes will diffuse longer distances before recombining as a direct result of the increased diffusivity.

\section{Conclusions}

The results for the first time demonstrate the dependence of interfacial carrier mobility on surface reactivity. It is proposed that the increased hole mobility at the n-GaN/aqueous interface results from water oxidation reaction intermediates and a local environment of hydroxylated and water adsorbed sites that leads to mobile hole hopping. Since oxidative catalysis proceeds from these reaction intermediates, the work suggests that surface reactivity and interfacial charge transport are dependent in the following sense: as a reaction intermediate is created that can transfer charge to reactant molecules, the speed with which a charge reaches a surface site increases. Future directions of this research will include investigating interfacial diffusivity not at open circuit, as done here, but while a steady catalytic current proceeds and measuring the affect of surface modifications (e.g. co-catalysts) on interfacial diffusivity. 


\section{Materials and Methods}

\section{Photo-electrochemistry}

Undoped and Si-doped GaN (0001) films on Cplane sapphire grown by hydride vapour phase epitaxy (HVPE)-based method were obtained from MTI Corporation (Richmond, CA). The GaN films are nominally $5 \mu \mathrm{m}$ thick.

A three-electrode customized Teflon electrochemical cell with $\mathrm{CaF}_{2}$ optical windows was used to observe TGS dynamics in situ. (Photo)Electrochemical measurements were carried out at room temperature with $\mathrm{Pt}$ counter electrode and a $\mathrm{Ag} / \mathrm{AgCl}$ reference electrode (MF-2052; Basi, West Lafayette, IN) using a CHI1140B Potentiostat (CH Instruments, Inc., Austin, TX) and constantly stirred. The electrolyte solution was $0.1 \mathrm{M}$ $\mathrm{HBr}$ and $0.1 \mathrm{M} \mathrm{Na}_{2} \mathrm{SO}_{4}$. Ohmic contacts were made with $\mathrm{Cu}$ wire and InGa eutectic with $\mathrm{Ag}$ paste on $\mathrm{n}-\mathrm{GaN}$. Insulating lacquer covered the contact to only expose $\sim 0.01 \mathrm{~cm}^{2}$ of $\mathrm{n}$ GaN. Cyclic voltammograms are provided in Supp. Fig. 10. Mott-Schottky plots yielded a doping density, $\mathrm{n}_{0}$, of $5 \times 10^{18} \mathrm{~cm}^{-3}$. Open circuit potential measurements were performed to determine photovoltage by illuminating with $0.06 \mathrm{~mJ} \mathrm{~cm}{ }^{-2}$ of a $266 \mathrm{~nm}$ pulsed light and/or continuous light from a Xe lamp (Supp. Fig. 10).

\section{Heterodyne Transient Optical Grating Spectroscopy}

Pump and probe beams were derived from a regeneratively amplified Ti:sapphire laser system (Coherent Legend; Coherent, Inc., Santa Clara, CA) producing output pulses with a center wavelength of $800 \mathrm{~nm}$ and $\sim 150$ fs width at a $1 \mathrm{kHz}$ repetition rate. Part of the amplifier output was frequency tripled using nonlinear crystals to create light at $266 \mathrm{~nm}$ for excitation above the band gap of GaN.

The transmitted and diffracted $800 \mathrm{~nm}$ probe beams were detected on two silicon photodiodes (DET36A; Thorlabs, Inc., Newton, NJ). In all experiments, the pump beam was modulated by a mechanical chopper (SR540; Stanford Re- search Systems, Inc., Sunnyvale, CA) at $300 \mathrm{~Hz}$. The detector output was digitized on a lock-in amplifier (SR810; Stanford Research Systems, Inc., Sunnyvale, CA) and interfaced with a personal computer, which provided automatized control over an optical pump-probe delay stage (MTM250CC1, Newport, Inc., Irvine, CA).

A diffractive optic was used to obtain the \pm 1 st order beams. Two time-coincident excitation pulses are crossed on the sample to produce an interference pattern. If the energy of the incident light coincides with an optical transition of the sample, the absorption mimics the interference pattern and a diffraction grating of excited states forms. The decay of the grating can, then, be probed by a third beam which gets diffracted by the induced grating. The intensity of the diffracted beam with respect to the time delay is proportional to $\Delta \mathrm{n}(\mathrm{t})^{2}$ where $\Delta \mathrm{n}(\mathrm{t})$ is the time dependent change in the index of refraction.

The diffracted signal was heterodyned by propagating pump and probe beams after the diffractive optic in a boxcar geometry and overlapping the probe and diffracted beam of opposite orders after the sample. ${ }^{42-45}$ The mixing of probe and diffracted beam results in a signal that is a convolution of the diffracted and probe electric fields.

$$
I_{\text {het }}=\left|E_{\text {probe }}\right|^{2}+\left|E_{\text {diff } f}\right|^{2}+E_{\text {probe }} E_{\text {diff } f} \cos (\phi)
$$

$\left|E_{\text {probe }}\right|^{2}$ represents the response of the system to the imaginary refractive index and is akin to a pump-probe signal. $\left|E_{\text {diff }}\right|^{2}$ is negligibly small compared to the other terms. The mixing term is modulated sinusoidally by the phase difference between the two probe beams, $\phi$. This was achieved through rotation of a coverslip automated with a motorized rotation platform (RP01, Thorlabs, Inc., Newton, NJ.). The amplitude of the sinusoidal signal as a function of the phase rotation yields the TGS signal at each time delay point, i.e. diffraction efficiency, $\eta(t)$.

Typical absorbed pump fluences were $\sim 0.06 \mathrm{~mJ} \mathrm{~cm} \mathrm{~cm}^{-2}$ with a pump spot size of $\sim$ $500 \mu \mathrm{m}$. These pump fluences correspond to carrier densities of $1 \times 10^{19} \mathrm{~cm}^{-3}$. 


\section{Acknowledgement}

Hoang Doan and Kevin Pollock were supported by the Air Force Office of Scientific Research under AFOSR Award No. FA9550-12-1-0337 during the completion of this work. Transient grating equipment was supplied by the Department of Energy Office of Basic Energy Sciences, under the CPIMS program KC030102 (FWP No. CH12CUK1). Finally, we thank Drs. Joseph Orenstein and James Hinton for helpful discussions.

\section{Supporting Information Available}

This information is available free of charge via the Internet at http://pubs.acs.org

\section{References}

(1) Bard, A.; Faulkner, L. Electrochemical Methods Fundamentals and Applications, 2nd ed.; John Wiley and Sons Ltd.: New York City, New York, 2002.

(2) Kautek, W.; Gerischer, H.; Tributsch, H. The Role of Carrier Diffusion and Indirect Optical Transitions in the Photoelectrochemical Behavior of Layer Type d-Band Semiconductors. J. Electrochem. Soc. 1980, 127, 2471-2478.

(3) Lin, Y.-G.; Hsu, Y.-K.; Basilio, A.; Chen, Y.-T.; Chen, K.-H.; Chen, L.C. Photoelectrochemical Activity on GaPolar and N-Polar GaN Surfaces for Energy Conversion. Opt. Express 2014, 22, A21-A27.

(4) Hwang, Y.; Wu, C.; Hahn, C.; Jeong, H.; Yang, P. Si/InGaN Core/Shell Hierarchical Nanowire Arrays and their Photoelectrochemical Properties. Nano Lett 2012, 12, 1678-1682.

(5) Kibria, M.; Nguyen, H.; Cui, K.; Zhao, S.; Liu, D.; Guo, H.; Trudeau, M.; Paradis, S.; Hakima, A.-R.; Mi, Z. One-Step Overall Water Splitting under Visible Light Using
Multiband InGaN/GaN Nanowire Heterostructures. ACS Nano 2013, 7, 78867893 .

(6) Wang, D.; Pierre, A.; Kibria, M.; Cui, K.; Han, X.; Bevan, K.; Guo, H.; Paradis, S.; Hakima, A.-R.; Mi, Z. WaferLevel Photocatalytic Water Splitting on GaN Nanowire Arrays Grown by Molecular Beam Epitaxy. Nano Lett. 2011, 11, 2353-2357.

(7) AlOtaibi, B.; Fan, S.; Vanka, S.; Kibria, M.; Mi, Z. A Metal-Nitride Nanowire Dual-Photoelectrode Device for Unassisted Solar-to-Hydrogen Conversion under Parallel Illumination. Nano Lett. 2015, 15, 6821-6828.

(8) Licht, S.; Wang, B.; Mukerji, S.; Soga, T.; Umeno, M.; Tributsch, H. Efficient Solar Water Splitting, Exemplified by $\mathrm{RuO}_{2}{ }^{-}$ Catalyzed AlGaAs/Si Photoelectrolysis. J. Phys. Chem. B 2000, 104, 8920-8924.

(9) Nakamura, A.; Ota, Y.; Koike, K.; Hidaka, Y.; Nishioka, K.; Sugiyama, M.; Fujii, K. A 24.4\% Solar to Hydrogen Energy Conversion Efficiency by Combing Concentrator Photovoltatic Modules and Electrochemical Cells. Appl. Phys. Exp. 2015, 8, 107101-4.

(10) Cui, Y.; Zhong, Z.; Wang, D.; Wang, W.; Lieber, C. High Performance Silicon Nanowire Field Effect Transistors. Nano Lett. 2003, 3, 149-152.

(11) Seo, M.; Dayeh, S.; Picraux, S.; Taylor, A.; Prasankumar, R. Mapping Carrier Diffusion in Single Silicon Core-Shell Nanowires with Ultrafast Optical Microscopy. Nano Lett. 2012, 12, 6334-6338.

(12) Upadhya, P.; Martinez, J.; Li, Q.; Wang, G.; Swartzentruber, B.; Taylor, A.; Prasankumar, R. Space-and-TimeResolved Spectroscopy of Single GaN Nanowires. Appl. Phys. Lett. 2015, 106, 263103-5. 
(13) Gutsche, C.; Niepelt, R.; Gnauck, M.; Lysov, A.; Prost, W.; Ronning, C.; Tegude, F.-J. Direct Determination of Minority Carrier Diffusion Lengths at Axial GaAs Nanowire p-n Junctions. Nano Lett. 2012, 12, 1453-1458.

(14) Ma, J.; Lee, W.; Bae, J.; Jeong, K.; Oh, S.; Kim, J.; Kim, S.-H.; Seo, J.-H.; Ahn, J.P.; Kim, H. et al. Carrier Mobility Enhancement of Tensile Strained Si and SiGe Nanowires via Surface Defect Engineering. Nano Lett. 2015, 15, 7204-7210.

(15) Gunawan, O.; Sekaric, L.; Majumdar, A.; Rooks, M.; Appenzeller, J.; Sleight, J.; Guha, S.; Haensch, W. Measurement of Carrier Mobility in Silicon Nanowires. Nano Lett. 2008, 8, 1566-1571.

(16) Niquet, Y.-M.; Delerue, C.; Krzeminski, C. Effects of Strain on the Carrier Mobility in Silicon Nanowires. Nano Lett. 2012, 12, 3545-3550.

(17) Fonoberov, V.; Balandin, A. Giant Enhancement of the Carrier Mobility in Silicon Nanowires with Diamond Coating. Nano Lett. 2006, 6, 2442-2446.

(18) Klahr, B.; Gimenez, S.; FabregatSantiago, F.; Hamann, T.; Bisquert, J. Water Oxidation at Hematite Photoelectrodes: The Role of Surface States. J. Am. Chem. Soc. 2012, 134, 4294-4302.

(19) Gomer, R. Surface Diffusion. Vacuum 1983, 33, 537-542.

(20) Valentin, C.; Selloni, A. Bulk and Surface Polarons in Photoexcited Anatase $\mathrm{TiO}_{2}$. J. Phys. Chem. Lett. 2011, 2, 2223-2228.

(21) Zhang, M.; de Respinis, M.; Frei, H. Time-Resolved Observations of Water Oxidation Intermediates on a Cobalt Oxide Nanoparticle Catalyst. Nat. Chem. 2014, 6, 362-367.

(22) Bard, A.; Bocarsly, A.; Fan, F.-R.; Walton, E.; Wrighton, M. The Concept of Fermi Level Pinning at Semiconductor/Liquid Junctions. Consequences for Energy Conversion Efficiency and Selection of Useful Solution Redox Couples in Solar Devices. J. Am. Chem. Soc. 1980, 102, 3671-3677.

(23) Bockris, J.; Khan, S. Surface Electrochemistry: A Molecular Level Approach, 2nd ed.; Plenum Press: New York City, New York, 1993; pp 59-202.

(24) Wronski, C.; Lee, S.; Hicks, M.; Kumar, S. Internal Photoemission of Holes and the Mobility Gap of Hydrogenated Amorphous Silicon. Phys. Rev. Lett. 1989, 63, 1420-1423.

(25) Šcăjev, P.; Jarašiūnas, K.; Okur, S.; Özgür, U.; Morkoç, H. Carrier Dynamics in Bulk GaN. J. Appl. Phys. 2012, 111, 023702-8.

(26) Malinauskas, T.; Jarašiūnas, K.; Miasojedovas, S.; Juršènas, S.; Beaumont, B.; Gibart, P. Optical Monitoring of Nonequilibrium Carrier Lifetime in Freestanding GaN by Time-Resolved Four-Wave Mixing and Photoluminescence Techniques. Appl. Phys. Lett. 2006, 88, 202109-3.

(27) Sjodin, T.; Li, C.-M.; Petek, H.; Dai, H.L. Ultafast Transient Grating Scattering Studies of Carrier Dynamics at a Silicon Surface. Chem. Phys. 2000, 251, 205-213.

(28) Jarasiunas, K.; Lovergine, N. Characterisation of Bulk Crystals and Structures by Light-Induced Transient Grating Technique. Mater. Sci. Eng. 2002, B91-92, 100-104.

(29) Hoffman, C.; Jarašiūnas, K.; Gerritsen, H.; Nurmikko, A. Measurement of Surface Recombination Velocity in Semiconductors by Diffraction from Picosecond Transient Free-Carrier Gratings. Appl. Phys. Lett. 1978, 33, 536-539.

(30) Kasinski, J.; Gomez-Jahn, L.; Faran, K.; Gracewski, S.; Dwayne Miller, R. Picosecond Dynamics of Surface Electron Trans- 
fer Processes: Surface Restricted Transient Grating Studies of the $\mathrm{n}-\mathrm{TiO}_{2} / \mathrm{H}_{2} \mathrm{O}$ Interface. J. Chem. Phys. 1989, 90, 12531269.

(31) Morishita, T.; Hibara, A.; Sawada, T.; Tsuyumoto, I. Ultrafast Charge Transfer at $\mathrm{TiO}_{2} / \mathrm{SCN}^{-}$(aq) Interfaces Investigated by Femtosecond Transient Reflecting Grating Method. J. Phys. Chem. B 1999, 103, 5984-5987.

(32) Nakabayashi, S.; Komuro, S.; Aoyagi, Y.; Kira, A. Transient Grating Method Applied to Electron-Transfer Dynamics at a Semiconductor/Liquid Interface. J. Phys. Chem. 1987, 91, 1696-1698.

(33) Gomez-Jahn, L.; Min, L.; Dwayne Miller, R. In Situ Picosecond Studies of Surface Reaction Dynamics at GaAs(100) Interfaces. Mol. Cryst. Liq. Cryst. 1991, 194, 181-190.

(34) Wang, D.; Buontempo, J.; Li, Z.; Miller, R. Picosecond Surface Restricted Grating Studies of n-GaAs (100) Liquid Junctions. Evidence for Interfacial Charge Transfer Approaching Adiabatic Limits. Chem. Phys. Lett. 1995, 232, 7-15.

(35) Chen, Y.-W.; Kuo, J.-L. Density Functional Study of the First Wetting Layer on the GaN (0001) Surface. J. Phys. Chem. C 2013, 117, 8774-8783.

(36) Chen, P.-T.; Sun, C.-L.; Hayashi, M. First-Principles Calculations of Hydrogen Generation Due to Water Splitting on Polar GaN Surfaces. J. Phys. Chem. C 2010, 114, 18228-18232.

(37) Kibria, M.; Zhao, S.; Chowdhury, F.; Wang, Q.; Nguyen, H.; Trudeau, M.; Guo, Z., H.and Mi Tuning the Surface Fermi Level on p-Type Gallium Nitride Nanowires for Efficient Overall Water Splitting. Nat. Commun. 2014, 5, 3825-6.

(38) Li, L.; Fan, S.; Mu, X.; Mi, Z.; Li, C.J. Photoinduced Conversion of Methane into Benzene over GaN Nanowires. J. Am. Chem. Soc. 2014, 136, 7793-7796.

(39) Kim, S.; Ebaid, M.; Kang, J.-H.; Ryu, S.W. Improve Efficiency and Stability of GaN Photoanode in Photoelectrochemical Water Splitting by $\mathrm{NiO}$ Cocatalyst. Appl. Surf. Sci. 2014, 305, 638-641.

(40) Schäfer, S.; Koch, A.; Cavallini, A.; Stutzmann, M.; Sharp, I. Charge Transfer Across the n-Type GaN-Electrolyte Interface. J. Phys. Chem. C 2012, 116, 2228122286 .

(41) Chakrapani, V.; Chandrashekhar, P.; Kash, K.; Anderson, A.; Sunkara, M.; Angus, J. Electrochemical Pinning of the Fermi Level: Mediation of Photoluminescence from Gallium Nitride and Zinc Oxide. J. Am. Chem. Soc. 2008, 130, 1294412952.

(42) Gedik, N.; Orenstein, J. Absolute Phase Measurement in Heterodyne Detection of Transient Gratings. Optics Letters 2004, 29, 2109-2111.

(43) Xu, Q.-H.; Ma, Y.-Z.; Fleming, G. Heterodyne Detected Transient Grating Spectroscopy in Resonant and Non-Resonant Systems using a Simplified Diffractive Optics Method. Chem. Phys. Lett. 2001, 338, 254-262.

(44) Goodno, G.; Dadusc, G.; Miller, R. Ultrafast Heterodyne-Detected TransientGrating Spectroscopy using Diffractive Optics. J. Opt. Soc. Am. B 1998, 15, 1791-1794.

(45) Maznev, A. A.; Nelson, K. A.; Rogers, J. A. Optical Heterodyne Detection of Laser-Induced Gratings. Optics Letters 1998, 23, 1319-1321.

(46) Fujii, K.; Ohkawa, K. Hydrogen Generation from Aqueous Water using n-GaN by Photoassisted Electrolysis. Phys. Stat. Sol. C 2006, 3, 2270-2273. 
(47) Huygens, I.; Strubbe, K.; Gomes, W. Electrochemistry and Photoetching of n-GaN. J. Electrochem. Soc. 2000, 147, 17971802 .

(48) Tseng, W.; van Dorp, D.; Lieten, R.; Mehta, B.; Vereecken, P.; Borghs, G. Enhanced Photocatalytic Activity of Nanoroughened GaN by Dry Etching. ECS Electrochem. Lett. 2013, 2, H51-H53.

(49) Nagasubramanian, G.; Wheeler, B.; Fan, F.-R.; Bard, A. XLII. Evidence for Fermi Level Pinning from Shifts in the Flatband Potential of p-Type Silicon in Acetonitrile Solutions with Different Redox Couples. J. Electrochem. Soc. 1982, 129, 1742-1745.

(50) Waegele, M.; Chen, X.; Herlihy, D.; Cuk, T. How Surface Potential Determines the Kinetics of the First Hole Transfer of Photo-Catalytic Water Oxidation. $J$. Am. Chem. Soc. 2014, 136, 10632-10639.

(51) Green, M. Electrochemistry of the Semiconductor-Electrolyte Electrode. I. The Electrical Double Layer. J. Chem. Phys. 1959, 31, 200-203.

(52) De Gryse, R.; Gomes, W.; Cardon, F.; Vennik, J. On the Interpretation of Mott-Schottky Plots Determined at Semiconductor/Electrolyte Systems. J. Electrochem. Soc. 1975, 122, 711-712.

(53) Uosaki, K.; Kita, H. Effects of the Helmholtz Layer Capacitance on the Potential Distribution at Semiconductor/Electrolyte Interface and the Linearity of the Mott-Schottky Plot. J. Electrochem. Soc. 1983, 130, 895-897.

(54) Srinivasan, S. Fuel Cells: From Fundamentals to Applications; Springer: New York City, New York, 2006.

(55) Laser, D.; Bard, A. Semiconductor Electrodes VII. Digital Simulation of Charge Injection and the Establishment of the Space Charge Region in the Absence and Presence of Surface States. J. Electrochem. Soc. 1976, 123, 1828-1832.

(56) Bermudez, V.; Long, J. Chemisorption of $\mathrm{H}_{2} \mathrm{O}$ on $\mathrm{GaN}(0001)$. Surf. Sci. 2000, 450, 98-105.

(57) Cooper, G.; Turner, J.; Parkinson, B.; Nozik, A. Hot Carrier Injection of Photogenerated Electrons at Indium PhosphideElectrolyte Interfaces. J. Appl. Phys. 1983, 54, 6463-6473.

(58) Turner, J.; Manassen, J.; Nozik, A. Photoelectrochemistry with p-Si Electrodes: Effects of Inversion. Appl. Phys. Lett. 1980, 37, 488-491.

(59) Schetzina, J.; McKelvey, J. Ambipolar Transport of Electrons and Holes in Anisotropic Crystals. Phys. Rev. B 1970, 2, 1869-1874.

(60) Aleksiejūnas, R.; Sūdžius, M.; Malinauskas, T.; Vaitkus, J.; Jarašiūnas, K.; Sakai, S. Determination of Free Carrier Bipolar Diffusion Coefficient and Surface Recombination Velocity of Undoped GaN Epilayers. Appl. Phys. Lett. 2003, 83, 1157-1159.

(61) Reshchikov, M.; Morkoç, H.; Park, S.; Lee, K. Yellow and Green Luminescence in a Freestanding GaN Template. Appl. Phys. Lett. 2001, 78, 3041-3043.

(62) Bandić, Z.; Bridger, P.; Piquette, E.; McGill, T. The Values of Minority Carrier Diffusion Lengths and Lifetimes in GaN and Their Implications for Bipolar Devices. Solid State Electron. 2000, 44, 221-228.

(63) Chernyak, L.; Osinsky, A.; Temkin, H.; Yang, J.; Chen, Q.; Khan, M. Electron Beam Induced Current Measurements of Minority Carrier Diffusion Length in Gallium Nitride. Appl. Phys. Lett. 1996, 69, 2531-2533.

(64) Chow, T. P.; Ghezzo, M. SiC Power Devices. Symposium E III-Nitride, SiC, and Diamond Materials for Electronic. 1996. 
(65) Kumakura, K.; Makimoto, T.; Kobayashi, N.; Hashizume, T.; Fukui, T.; Hasegawa, H. Minority Carrier Diffusion Length in GaN: Dislocation Density and Doping Concentration Dependence. Appl. Phys. Lett. 2005, 86, 052105-3.

(66) Kozodoy, P.; Xing, H.; DenBaars, S.; Mishra, U.; Saxler, A.; Perrin, R.; Elhamri, S.; Mitchel, W. Heavy Doping Effects in Mg-Doped GaN. J. Appl. Phys. 2000, 87, 1832-1835.

(67) Zhong, Z.; Qian, F.; Wang, D.; Lieber, C. Synthesis of p-Type Gallium Nitride Nanowires for Electronic and Photonic Nanodevices. Nano Lett. 2003, 3, 343346.

(68) Liaugaudas, G.; Dargis, D.; Kwasnicki, P.; Arvinte, R.; Zielinski, M.; Jarašiūnas, K. Determination of Carrier Lifetime and Diffusion Length in Al-Doped 4H-SiC Epilayers by Time-Resolved Optical Techniques. J. Phys. D: Appl. Phys. 2015, 48, 025103-7.

(69) Linnros, J.; Grivickas, V. CarrierDiffusion Measurements in Silicon with a Fourier-Transient-Grating Method. Phys. Rev. B 1994, 50, 16943-16955.

(70) Jarašiūnas, K.; Šcăjev, P.; Nargelas, S.; Malinauskas, T.; Kato, M.; Ivakin, E.; Nesladek, M.; Haenen, K.; Özgür, U.; Morkoç, H. Recombination and Diffusion Processes in Polar and Nonpolar Bulk GaN Investigated by Time-Resolved Photoluminescence and Nonlinear Optical Techniques. Mat. Sci. Forums 2012, 717720, 309-312.

(71) Tseng, W.; van Dorp, D.; Lieten, R.; Vereecken, P.; Borghs, G. Anodic Etching of n-GaN Epilayer into Porous GaN and Its Photoelectrochemical Properties. J. Phys. Chem. C 2014, 118, 2949229498.

(72) Fujii, K.; Karasawa, T.; Ohkawa, K. Hydrogen Gas Generation by Splitting Aque- ous Water Using n-Type GaN Photoelectrode with Anodic Oxidation. Jap. J. Appl. Phys. 2005, 44, L543-L545.

(73) Ye, H.; Chen, G.; Niu, H.; Zhu, Y.; Shao, L.; Qiao, Z. Structures and Mechanisms of Water Adsorption on $\mathrm{ZnO}(0001)$ and GaN(0001) Surface. J. Phys. Chem. C 2013, 117, 15976-15983.

(74) Akimov, A.; Muckerman, J.; Prezhdo, O. Nonadiabatic Dynamics of Positive Charge During Photocatalytic Water Splitting on GaN(10-10) Surface: Charge Localization Governs Splitting Efficiency. J. Am. Chem. Soc. 2013, 135, 8682-8691.

(75) Paul, D.; Mitra, S. Evaluation of Mott's Parameters for Hopping Conduction in Amorphous Ge, Si, and Se-Si. Phys. Rev. Lett. 1973, 31, 1000-1003.

(76) Ambegaokar, V.; Halperin, B.; Langer, J. Hopping Conductivity in Disordered Systems. Phys. Rev. B 1971, 4, 2612-2620. 
